\title{
Analysis of 3D Magnetic Field of Magnetic Suspension Spherical Reluctance Motor
}

\author{
Hu Daotian \\ College of Mechanical Engineering Yangzhou University \\ Yangzhou University,YZU \\ Yangzhou, China,18252718210 \\ tian3.happy@163.com
}

\begin{abstract}
It is a very complicated problem that analyzing and calculating the magnetic field of the magnetic suspension spherical reluctance motor, because the structure of the stator and rotor, the distribution of magnetic field present three-dimension. By calculating 3-D magnetic field and analyzing the magnetic field of the magnetic suspension spherical reluctance motor, Then we draw the magnetic flux density distribution curve of the salient pole stator and rotor in different position, when the motor stator is in different current.
\end{abstract}

Keywords-magnetic suspension; finite element method; $3 D$ magnetic field; spherical reluctance motor

\section{INTRODUCTION}

Multiple degrees of freedom motor refers to a kind of motor which has two or three rotating degrees, the motor can rotate round fixed-point axis of the space. The spherical motor belongs to multiple degrees of freedom (MDOF) motor. MDOF motor has a wide application prospect in robots, multiple coordinates mechanical processing center, aerospacecraft, electric gyroscope, all-round tracking antenna, turret turntable, prosthetic human body, medical equipment, camera work station, panorama photography work station, blender, globe valve, and other multiple degrees of freedom equipments. ${ }^{[1]}$ It is too difficult to simplify magnetic field of spherical motor by using 2D magnetic field because it has complex boundary conditions, anisotropy, nonlinearity and other characteristics, and that it is a typical 3D magnetic field, we must further discuss its calculation problem. Currently, the subject about the design and analysis of spherical motor is still being studied at home and abroad. ${ }^{[2-3]}$ This study plans to use 3D finite element analysis(FEA)method to calculate and analyze the magnetic field of MDOF magnetic suspension spherical reluctance motor in order to offer some basis for the design and calculation of MDOF magnetic suspension spherical reluctance motor.

\section{The BASIC Structure OF MAgnetic SUSPENSION SPHERICAL RELUCTANCE MOTOR}

The stator and rotor 3D structure of magnetic suspension spherical reluctance motor are shown in Fig.1,the rotor is a unit salient pole sphere that has grooves, and it locates in the middle of the motor; Four stators are symmetrically distributed on the

The China Natural Science Foundation for this project. Item Number: 50975249.and Natural Science Foundation of Universities in Jiangsu Pro for this project. Item Number: 08KJB460008.

\author{
Zeng Li \\ College of Mechanical Engineering Yangzhou University \\ Yangzhou University,YZU \\ Yangzhou, China,13665203656 \\ lizengcf@163.com
}

equator line of spherical rotor. stator 1 and stator 3 keep coaxial with the $\mathrm{x}$-axis; the function is to drive the rotor to rotate around $\mathrm{x}$-axis and produce magnetic suspension force that makes the rotor stabilize in the $\mathrm{x}$-axis direction, Stator 2 and stator 4 keep coaxial with the y-axis, which is aimed at driving the rotor to rotate around the $y$-axis and producing magnetic suspension force that makes the rotor stabilize in the y-axis direction; Stator 5 is located in the top of spherical rotor and keeps coaxial with $\mathrm{z}$-axis, its function is to drive the spherical rotor to rotate around z-axis and produce stable magnetic suspension force in the $\mathrm{z}$-axis direction.

The surface of spherical rotor of magnetic suspension spherical motor has mutual orthogonal grooves. The stator includes six salient poles and one ladder circle. Based on reluctance motor principle, there are multiphase windings which drive the stator to rotate in salient poles of stator. After each phase winding is charged with electricity, it not only produces electromagnetic torque which drives the rotor to rotate, but also provides radial magnetic suspension force for rotor. ${ }^{[4]}$

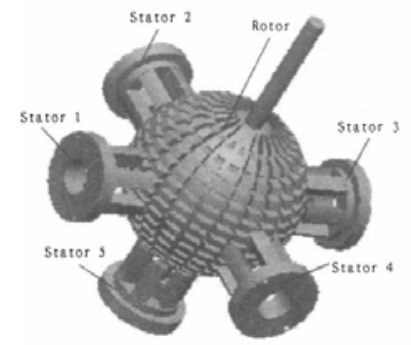

Fig. 1 The structure of the magnetic suspension spherical reluctance motor

\section{ANALYSIS OF MAGNETIC FIELD OF MAGNETIC SUSPENSION SPHERICAL RELUCTANCE MOTOR}

\section{A. The Basic Principle of 3D Magnetic Field Calculation of} Magnetic Suspension Spherical Reluctance Motor

In the finite element of $3 \mathrm{D}$ magnetic field, three components of the magnetic vector potential $A$ are $A_{x}, A_{y}, A_{z}$. ${ }^{[5-6]}$ In the magnetic field of $3 \mathrm{D}$ linear media, the magnetic vector potential A satisfy following demands under $\nabla \bullet A=0$ :

$\nabla^{2} A=-\mu J$,In the magnetic field $\mathrm{V}$; 
$A /{ }_{s}=C$, on the boundary S;

Corresponding conditions variational problem is: $F(A)=\iiint\left[\frac{1}{2 \mu}|\nabla \times A|^{2}-J \cdot A\right] d x d y d z=\min ,\left.A\right|_{s}=C$

If the whole magnetic field is discrete into $\mathrm{k}_{0}$ units and $\mathrm{p}_{0}$ nodes. In the rectangular coordinate system,the magnetic vector potential $A_{i}$ of any node $i$ has three components, which are called $A_{x i}, A_{y i}, A_{z i}$; Therefore,each component of the magnetic vector potential of $\mathrm{p}_{0}$ nodes can be lined into the following column vector:

$$
[A]=\left[A_{x 1} A_{y 1} A_{z 1} A_{x 2} \ldots A_{x p}\right]
$$

If each unit has $\mathrm{p}_{0}$ nodes,the shape function corresponding to node i is $P_{i}^{k}$, and the magnetic vector potential A of any point of each unit can be expressed as $A=\sum_{i=1}^{p_{0}} P_{i}^{k} A_{i}$. Three components of the magnetic vector potential $A$ are respectively expressed as

$$
A_{x}=\sum_{i=1}^{p_{0}} P_{i}^{k} A_{x i}, A_{y}=\sum_{i=1}^{p_{0}} P_{i}^{k} A_{y i}, A_{z}=\sum_{i=1}^{p_{0}} P_{i}^{k} A_{z i}
$$

Energy functional $\mathrm{F}(\mathrm{A})$ of the whole magnetic field can be expressed as the sum of each unit energy functional: $F(A) \approx F(\bar{A})=\sum_{i=1}^{k_{0}} F_{k}(\bar{A})$

The unit energy functional is

$$
\begin{aligned}
& F_{k}(A)=\iiint_{k}\left[\frac{1}{2 \mu}|\nabla \times A|^{2}-J \cdot A\right] d x d y d z=\iiint_{k}\left\{\frac{1}{2 \mu}\right. \\
& {\left[\left(\frac{\partial A_{z}}{\partial y}-\frac{\partial A_{y}}{\partial z}\right)^{2}+\left(\frac{\partial A_{x}}{\partial z}-\frac{\partial A_{z}}{\partial x}\right)^{2}+\left(\frac{\partial A_{y}}{\partial x}-\frac{\partial A_{x}}{\partial y}\right)^{2}\right]} \\
& \left.-\left(J_{x} A_{x}+J_{y} A_{y}+J_{z} A_{z}\right)\right\} d x d y d z
\end{aligned}
$$

According to extreme value theory,the variational problem is equivalent to the following equation:

$$
\begin{aligned}
& \frac{\partial F}{\partial A_{x i}}=\sum_{k=1}^{k_{0}} \frac{\partial F_{k}}{\partial A_{x i}}=0, \frac{\partial F}{\partial A_{y i}}=\sum_{k=1}^{k_{0}} \frac{\partial F_{k}}{\partial A_{y i}}=0, \\
& \frac{\partial F}{\partial A_{z i}}=\sum_{k=1}^{k_{0}} \frac{\partial F_{k}}{\partial A_{z i}}=0
\end{aligned}
$$

In the above equation, any unit $\mathrm{k}$ after discretization has $\mathrm{p}_{0}$ nodes. Then the ith node of this unit can be showed as

$$
\begin{aligned}
& \frac{\partial F_{k}}{\partial A_{x i}}=\sum_{j=1}^{p_{0}} \iiint_{k}\left\{\frac { 1 } { \mu } \left[\left(\frac{\partial P_{i}^{k}}{\partial y} \frac{\partial P_{j}^{k}}{\partial y}+\frac{\partial P_{i}^{k}}{\partial z} \frac{\partial P_{j}^{k}}{\partial z}\right) A_{x j}-\right.\right. \\
& \left.\left.\left(\frac{\partial P_{j}^{k}}{\partial x} \frac{\partial P_{i}^{k}}{\partial y}\right) A_{y i}-\left(\frac{\partial P_{j}^{k}}{\partial x} \frac{\partial P_{i}^{k}}{\partial z}\right) A_{z j}\right]-J_{x} N_{i}^{k}\right\} d x d y d z \\
& \frac{\partial F_{k}}{\partial A_{y i}}=\sum_{j=1}^{p_{0}} \iiint_{k}\left\{\frac { 1 } { \mu } \left[\left(\frac{\partial P_{i}^{k}}{\partial x} \frac{\partial P_{j}^{k}}{\partial x}+\frac{\partial P_{i}^{k}}{\partial z} \frac{\partial P_{j}^{k}}{\partial z}\right) A_{y j}-\right.\right. \\
& \left.\left.\left(\frac{\partial P_{i}^{k}}{\partial x} \frac{\partial P_{j}^{k}}{\partial y}\right) A_{x j}-\left(\frac{\partial P_{j}^{k}}{\partial y} \frac{\partial P_{i}^{k}}{\partial z}\right) A_{z j}\right]-J_{y} N_{i}^{k}\right\} d x d y d z \\
& \frac{\partial F_{k}}{\partial A_{z i}}=\sum_{j=1}^{p_{0}} \iiint_{k}\left\{\frac { 1 } { \mu } \left[\left(\frac{\partial P_{i}^{k}}{\partial x} \frac{\partial P_{j}^{k}}{\partial x}+\frac{\partial P_{i}^{k}}{\partial z} \frac{\partial P_{j}^{k}}{\partial z}\right) A_{z j}-\right.\right.
\end{aligned}
$$

$\left.\left.\left(\frac{\partial P_{i}^{k}}{\partial x} \frac{\partial P_{j}^{k}}{\partial z}\right) A_{x j}-\left(\frac{\partial P_{j}^{k}}{\partial y} \frac{\partial P_{i}^{k}}{\partial z}\right) A_{y j}\right]-J_{y} N_{i}^{k}\right\} d x d y d z$

Note that its value is not zero only when $i$ is the node of this unit; Therefore, unit coefficient matrix corresponding to one unit is $[S]_{k}=\left.\left[\left[S_{i j}\right]_{k}\right] S_{i j}\right|_{k}$, among the equation is third matrix. $[S]_{k}$ is composed of $\left[S_{i j}\right\rfloor_{k}$ square matrix that the number is $p_{0} \times p_{0}$.The same argument, $[N]_{k}=\left[N_{x 1}^{k} N_{y 1}^{k} N_{z 1}^{k} \ldots N_{x p_{0}}^{k} N_{y p_{0}}^{k} N_{z p_{0}}^{k}\right]^{T}$, among the equation,

$$
\begin{aligned}
& N_{x i}^{k}=\iiint_{v_{k}} J_{x} P_{j}^{k} d x d y d z, \quad N_{y i}^{k}=\iiint_{v_{k}} J_{y} P_{j}^{k} d x d y d z, \\
& N_{z i}^{k}=\iiint_{v_{k}} J_{z} P_{j}^{k} d x d y d z, i=1,2, \ldots, p_{0} .
\end{aligned}
$$

We should solve the coefficient matrix of above unit, then realize further expansion , getting the finite element equation that is $[S][A]=[N]$. Solution of above equation is [A].

\section{B. Establishment of Model and the Decision of Boundary Condition}

Numerical analysis for electromagnetic field of motor mainly use the finite element method that is the most effective and the most widely used at present, boundary element method, and finite difference method. ANSYS is a kind of finite element analysis software ,which is widely used at present. ${ }^{[7]}$ The basic principle of analysis for electromagnetic field using ANSYS is that processing object first should be divided into finite unit(including some nodes),then magnetic potential or electric potential of the each node under certain boundary conditions and initial conditions can be calculated according to magnetic vector potential and electric scalar potential.

TAB.1 THE STRUCTURAL PARAMETERS OF MAGNETIC SUSPENSION SPHERICAL RELUCTANCE MOTOR

\begin{tabular}{|c|c|}
\hline the radius of spherical top of stator salient pole/mm & 51 \\
\hline unilateral air gap of between stator surface and rotor surface/mm & 1 \\
\hline the radius of outside spherical surface of rotor/mm & 50 \\
\hline the radius of inner spherical surface of stator/mm & 20 \\
\hline the radius of outside spherical surface of stator/mm & 38 \\
\hline the length of air gap/mm & \\
\hline the number of stator groove & 1 \\
\hline the number of groove in rotor latitude direction & 7 \\
\hline the number of groove in rotor longitude direction & 12 \\
\hline the number of stator coil & 50 \\
\hline polar altitude of stator/mm & 25 \\
\hline coil current/A & I \\
\hline
\end{tabular}

Based on magnetic potential and electric potential, we can get other physical quantities of electric field such as the 
distribution of line of magnetic force, magnetic induction, electric displacement flux, energy of electromagnetic field, magnetic force, torque, inductance, capacitance and so on through dealing with the solution. Due to the symmetry of structure of magnetic suspension spherical reluctance motor ,only one stator and rotor are choosed to research and start the model.The structural parameters of magnetic suspension spherical reluctance motor are showed in Table 1. The grid of motor is showed in Fig.2(there are no air and winding parts ).The grid pattern of rotor is partly showed in Fig.3.

In Fig. 2 and 3,the unit after meshing of solid modeling is showed. Thanks to having magnetic leakage in air, the practical calculation is performed outside the whole model including the air.

All physical units in model choose tetrahedral unit SOLID98 having 10 nodes(with a view to geometry irregularity of magnetic suspension spherical reluctance motor ), and boundary units of the air choose tetrahedral unit INFIN47 having four nodes. ${ }^{[8]}$ When meshing, it should be taken into account that air gap between stator and rotor is a position that the variation of magnetic field is large, so grid density should be relatively close. Considering geometry symmetry of motor, both sides of stator salient pole satisfy half a cycle boundary conditions in the range of the stator and rotor relative a polar distance. On other boundary face, lines of magnetic force and boundary surface is considered to be parallel and internal boundary conditions of motor can meet itself. $^{[9]}$

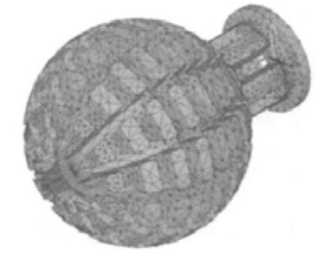

Fig.2 Motor meshing map

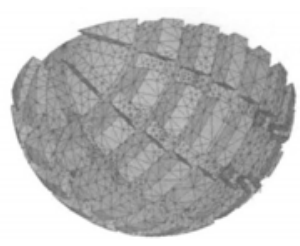

Fig.3 Rotor meshing map

\section{Calculated Results}

By using ANAYS, a plane where it is in the air gap between rotor salient pole and stator salient pole twined by coils is intercepted, then we can figure out magnetic flux through this plane. ${ }^{\left[10^{-11]}\right.}$ In this paper, we will figure out the magnetic flux through this plane, when the rotor of magnetic suspension spherical motor per rotates $1^{\circ}$ around z-axis. ${ }^{[12-13]}$

It is shown in Fig.4 that variation curves of the magnetic flux density of the section which is in the air gap between part of the stator pole and rotor salient pole along with the variation of rotor rotation angle in different stator current, The paper sets the situation that stator salient pole axis coincides with rotor concave pole axis to $0^{\circ}$.In Fig.4, when stator salient pole axis coincides with rotor concave pole axis, the number of magnetic flux produced by stator core which goes into rotor salient pole magnetism gathering structure is largest, at this moment, magnetic flux density of the air gap is largest. With rotor salient pole straying from the central position of stator salient pole axis, the leakage magnetic flux of the motor becomes larger, and the magnetic flux density of section where it is in the air gap becomes smaller and smaller. But when it enters into the next magnetic pole, the magnetic flux density of section starts to become larger again.
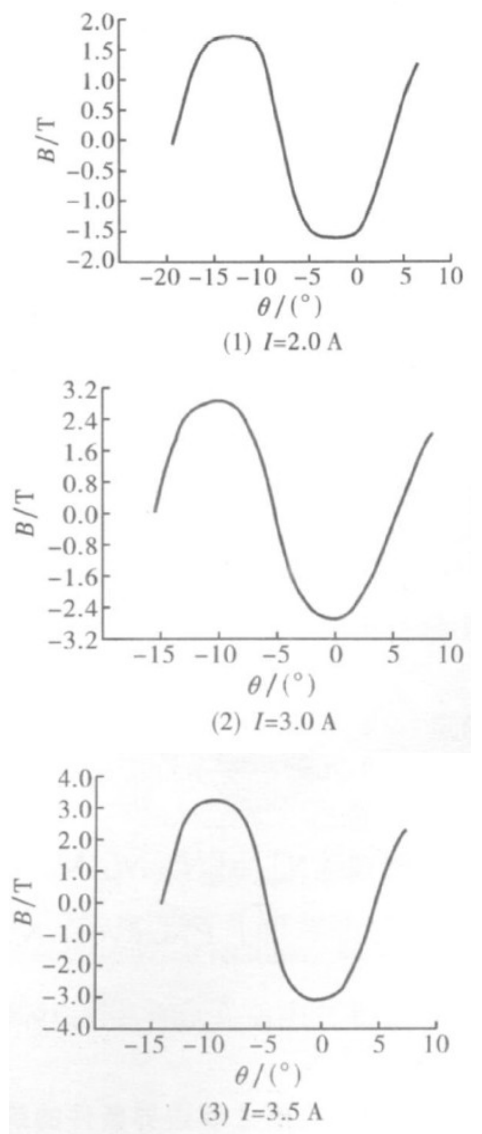

Fig.4 Magnetic flux density curve

\section{REFERENCES}

[1] Shenghua Huang. 3D motor and control system[M].Wuhan:Huazhong University of Science and Technology Press,1988:1-11.

[2] M.Ashimot, T.Oyamas, I.Shidah. Design and implementation of spherical ultrasonic motor[J].IEEE Trans Ultrason Ferroelectr Freq Control, 2009, 56(11):2514-2521

[3] Zhanghe Wang, Li Zheng, Mingming Jiang.Current development situation and research direction of MDOF spherical joint motor[J].Modern Manufacturing Engineering, 2010(1):145-149.

[4] Xiuhong Zhou, Li Zheng. Basic principle and key technology of magnetic suspension spherical reluctance motor[J].Academic Journal of Xuzhou Institute of Technology, 2007, 22(8):72-75.

[5] Xiaohua Bao, Qunjing Wang, Youyuan Ni, et al. Efficient electromagnetic modeling analysis based on supporting automotive claw-pole alternator of vector machine $[\mathrm{J}]$. Automotive engineering,2006,28(2):186 -189.

[6] Xiaohua Bao, Qunjing Wang, Youyuan Ni, et al.Modeling and optimized parameter design of claw-pole alternator[J].Proceedings of the CSEE,2006,26(21):138 -142.

[7] Qunjing Wang, Li Zheng, Youyuan Ni, et al. 3Dmagnetic field analysis and torque calculation of a PM spherical motor[C]. Proceeding of the Eighth International Conference on Electrical Machines and Systems. Nanjing:[s.n.], 2005,3:2116-2120. 
[8] O.Kaday, Y.Amashiron, Ohmorik, et al. Mixed flow artificial heart pump with axial self-bearing motor[J]. IEEE/ASME Trans Mechatronics, 2005, 10(6):658-665.

[9] Zhaowen Yan, Xianghui Wang, Qiebing Wang, et al. ANSYS 10.0 engineering electromagnetic analysis technique and example explanation[M].Beijing:China Water Resources and Electricity Press,2006:215-292.

[10] O.hamorik, K.imsj, M.asuzawat, et al. Design of an axial-type self-bearing motor for small axial pump[C].8th International Symposium on Magnetic Bearing. Mito, Japan: [s.n.], 2002:15-20.
[11] D.aveyk, V.achtsevanosg, P.owersr. The analysis of fields and torques in spherical induction motors[J].IEEE Trans on Magn, 1987, 23(1):273-282.

[12] Jinghua Ji, Yuhun Sun, Huangqiu Zhu, et al. Magnetic field analysis of bearing switched reluctance motor using finite element mothod[J]. Proceeding of the Eighth International Conference on Electrical Machines and Systems. Nanjing:[s.n.],2005,3:2121-2123.

[13] Changliang Xia, Hongfeng Li, Tingna Shi. 3D magnetic field and torque analysis of a novel Halbach array permanent magnet spherical motor[J].IEEE Trans on Magn, 2008, 44(8):2016-2020. 